\title{
Focused sticking of light mass particles in physisorption
}

\author{
S. Miret-Artés \\ Instituto de Matemáticas y Física Fundamental, Consejo Superior de Investigaciones Científicas, Serrano, 123, 28006 Madrid, Spain \\ J. R. Manson \\ Department of Physics and Astronomy, Clemson University, Clemson, South Carolina 29634
}

(Received 2 August 2000; published 7 March 2001)

\begin{abstract}
In the scattering of atoms and molecules from surfaces, focusing effects can give rise to a variety of significant features in both the experimentally measured elastic and inelastic scattering intensities. The study of focusing is extended to the case of sticking of atoms upon scattering into the physisorption potential well. It is demonstrated that this focused sticking (FS) should give rise to enhancements of the sticking coefficient for certain well-defined incident conditions.
\end{abstract}

DOI: 10.1103/PhysRevB.63.121404

PACS number(s): 82.65.+r, 68.43.Mn, 79.20.Rf

When a well-defined beam of atomic or molecular projectiles is directed towards a surface, typically a fraction of the beam will be backscattered into outgoing continuum states and the remaining fraction will become trapped in the physisorption well of the interaction potential. The trapping in the physisorption well may be transient, with further rapid transition into a positive energy outgoing continuum state, or the projectile may make transitions leading to a state of negative total energy and become adsorbed at the surface. The fraction of incoming particles that adsorb on a clean surface is the sticking coefficient.

In the last two decades, the role of resonance processes leading to sticking in the physisorption well has been examined in a large number of papers, both experimentally and theoretically as has been chronicled in a recent comprehensive review, see Ref. 1. Entry into the bound states can be via an elastic channel or via a phonon-mediated (inelastic) channel. The sticking coefficient often displays a characteristic feature whenever the incident scattering parameters, e.g., the energy and angles, fulfill the conditions for a selective adsorption process, either elastic or inelastic. ${ }^{2-5}$ However, the sticking is not a state-to-state transition, rather it must be regarded as a global property of the system because it involves a summation over all elementary processes that can lead to eventual adsorption.

During this same period of time, a number of resonance and focusing enhancement mechanisms have been predicted $^{6-9}$ for the case of scattering into continuum states, and some of them have been observed experimentally in either the scattering of He atom beams, or in the scattering of small mass molecular beams. ${ }^{10-12}$ The best known of the resonance effects is selective adsorption, which gives rise to sharp features in the elastic scattering intensities, ${ }^{13}$ and when assisted by a phonon transfer can give rise to significant enhancements of the energy-resolved inelastic scattering intensity. ${ }^{10}$ Focusing effects, on the other hand, represent a different type of enhancement, one in which the incident beam wave packet is sharply focused into a particular transition.

Up until now, focusing processes have been extensively exploited in continuum state surface scattering ${ }^{11,12}$ but their role in scattering into bound states and in sticking has not been investigated. The purpose of this paper is to point out conditions, called focused sticking (FS), under which focusing effects can significantly enhance the sticking coefficient of atomic and molecular projectiles.

For any atom-surface scattering event on a periodic surface the kinematical constraints are conservation of total energy $\Delta E$ and parallel momentum $\Delta K$ which are expressed as

$$
\Delta E=k_{i}^{2}-k_{f}^{2},
$$

and

$$
\Delta K=k_{f} \sin \theta_{f}-k_{i} \sin \theta_{i},
$$

where $\mathbf{k}_{i}$ and $\mathbf{k}_{f}$ are the incident and final wave vectors, $\theta_{i}$ and $\theta_{f}$ are the corresponding scattering angles relative to the surface normal, and the dimensions are chosen so that $\hbar^{2} / 2 m=1$ where $m$ is the projectile mass. Equation (2) is written for the special case of scattering in the sagittal plane (the plane defined by the incident beam and the surface normal), however, the extension to full three-dimensional scattering is straightforward.

In the case of scattering into a bound state of the physisorption potential labeled by the quantum number $n$, the final energy is given by $k_{f}^{2}=-\left|\epsilon_{n}\right|+\left(\Delta K+N+k_{i} \sin \theta_{i}\right)^{2}$ where $N$ is a surface reciprocal lattice vector and $\epsilon_{n}$ is the bound-state energy. Equations (1) and (2) can then be combined into the so-called resonance curve equation

$$
\Delta E=k_{i}^{2}-\left(k_{i} \sin \theta_{i}+N+\Delta K\right)^{2}+\left|\epsilon_{n}\right| .
$$

Equation (3) represents the locus, for a given set of incident conditions $\left(\theta_{i}\right.$ and $k_{i}^{2}$ ), of all elastic and inelastic processes compatible with the conservation rules in the dispersion $(\Delta E, \Delta K)$ space. Thus, crossings of these curves with the dispersion curves of phonons give rise to inelastic features in angular distributions and/or in time-of-flight inelastic intensity spectra. Conversely, if the energy $\Delta E$ and parallel momentum $\Delta K$ of the phonon are regarded as fixed, then the incident parameters $k_{i}$ and $\theta_{i}$ are consequently related by Eq. (3).

The total sticking coefficient from a given incident beam, regarded as a plane wave of wave vector $\mathbf{k}_{i}$, is the sum over 
all possible final states that can leave a particle adsorbed on the initially zero-coverage surface:

$$
\sigma\left(\mathbf{k}_{i}\right)=\sum_{N, \Delta E, \Delta K} \sigma\left(\mathbf{k}_{i} ; n, N, \Delta E, \Delta K\right)
$$

where $\sigma\left(\mathbf{k}_{i} ; n, N, \Delta E, \Delta K\right)$ can be regarded as the transition probability or the partial sticking coefficient into each welldefined quantum state, and the sum over $\Delta E$ includes the sum over all bound states $n$. In a real experiment, the incident beam is not a plane wave but a wave packet, and Eq. (4) must be convoluted over the distribution function $\rho_{\mathbf{k}_{i 0}}\left(\mathbf{k}_{i}\right)$ of the incident beam, where $\mathbf{k}_{i 0}$ is the central wave vector of the incident wave packet distribution. Thus the experimentally measured sticking coefficient $\Sigma\left(\mathbf{k}_{i 0}\right)$ is given by

$$
\Sigma\left(\mathbf{k}_{i 0}\right)=\int_{\Delta k_{i}} d k_{i} \int_{\Delta \theta_{i}} d \theta_{i} \rho_{\mathbf{k}_{i 0}}\left(\mathbf{k}_{i}\right) \sigma\left(\mathbf{k}_{i}\right) .
$$

$\Sigma\left(\mathbf{k}_{i 0}\right)$ can also be written as a sum over the individual quantum transition probabilities $\Sigma\left(\mathbf{k}_{i 0} ; n, N, \Delta E, \Delta K\right)$ just as for $\sigma\left(\mathbf{k}_{i}\right)$ in Eq. (4).

The question of focusing becomes of importance when the properties of the incident beam distribution are examined. Suppose that the incident beam has an angular spread but the incident energy is well defined at each angle within this angular spread. In such a case the incident distribution function can be reasonably approximated by

$$
\rho_{\mathbf{k}_{i 0}}\left(\mathbf{k}_{i}\right)=\delta\left(k_{i}-k_{i}\left(\theta_{i}\right)\right) g\left(\theta_{i}\right) .
$$

The result of carrying out the integral over the angular spread of the incident beam as in Eq. (5) for each quantum state component is the following:

$$
\begin{aligned}
& \sum\left(\mathbf{k}_{i 0} ; n, N, \Delta E, \Delta K\right) \\
& \quad=\int_{\Delta k_{i}} d k_{i}\left|\frac{d \theta_{i}}{d k_{i}}\right|_{\theta_{i}^{*}} g\left(\theta_{i}^{*}\right) \sigma\left(\mathbf{k}_{i} ; n, N, \Delta E, \Delta K\right),
\end{aligned}
$$

where $\theta_{i}^{*}\left(k_{i}\right)$ is the value given by the resonance equation (3) for specified $\Delta E$ and $\Delta K$.

The Jacobian derivative appearing in Eq. (7) can be readily calculated for fixed $\Delta E$ and $\Delta K$ and is

$$
\frac{d \theta_{i}}{d k_{i}}=\frac{k_{i} \cos ^{2} \theta_{i}-(\Delta K+N) \sin \theta_{i}}{k_{i} \cos \theta_{i}\left(k_{i} \sin \theta_{i}+\Delta K+N\right)} .
$$

The phenomenon of focusing occurs when this derivative is singular, and clearly this can occur when the denominator vanishes. In order to exhibit this singular behavior it is useful to solve Eq. (3) for $\theta_{i}$ as a function of $k_{i}$

$$
\theta_{i}=\arcsin \left(\frac{\sqrt{k_{i}^{2}-\Delta E+\left|\epsilon_{n}\right|}-\Delta K-N}{k_{i}}\right) .
$$

This angle must be real and in the range $-90^{\circ}<\theta_{i}<90^{\circ}$. Thus the minimum value of $\theta_{i}$ occurs for

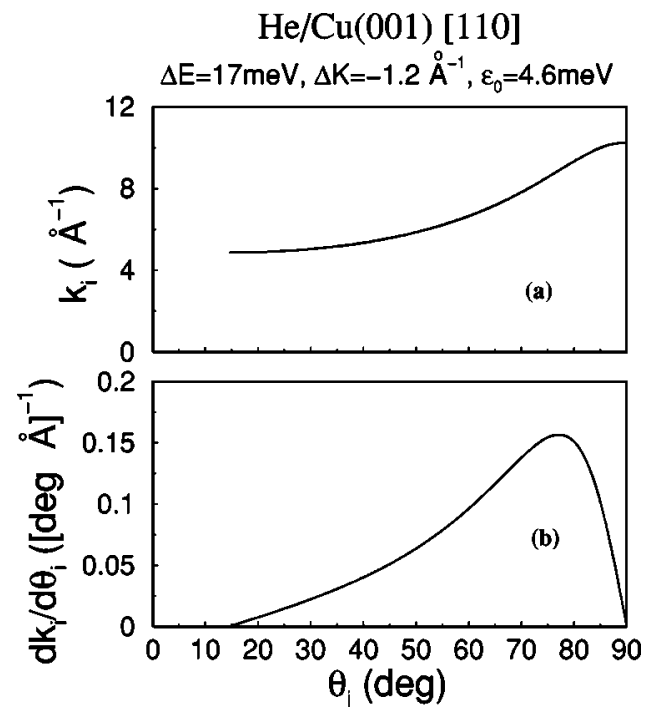

FIG. 1. Calculations demonstrating the focusing conditions for the case of $\Delta E=17 \mathrm{meV}$ and $\Delta K=-1.2 \AA^{-1}, N=0$, and $\epsilon_{0}$ $=4.6 \mathrm{meV}$, corresponding approximately to He scattering from $\mathrm{Cu}(001)<110>$ and a phonon at the Brillouin zone boundary: (a) $k_{i}$ as a function of $\theta_{i}$ from Eqs. (3) or (9), and (b) $d k_{i} / d \theta_{i}$ as a function of $\theta_{i}$, from Eq. (8).

$$
\theta_{\min }=\arcsin \left(\frac{-\Delta K-N}{\sqrt{\Delta E-\left|\epsilon_{n}\right|}}\right),
$$

at the corresponding minimum value of $k_{i}$ given by

$$
k_{\min }=\sqrt{\Delta E-\left|\epsilon_{n}\right|} .
$$

It is readily shown that the Jacobian derivative of Eq. (8) is singular at this minimum point due to the fact that $k_{\min } \sin \theta_{\min }+\Delta K+N=0$. Thus the incident angle and wave vector determined by $\theta_{\min }$ and $k_{\min }$ are the conditions for focusing in the sticking process.

A second condition for focusing is found in the case in which $\Delta K+N<0$ at $\theta_{\max }=90^{\circ}$ with the corresponding value of $k_{i}=k_{\max }$ given by

$$
k_{\max }=\frac{\left|\epsilon_{n}\right|-\Delta E-(\Delta K+N)^{2}}{2(\Delta K+N)} .
$$

This is the case of a beam incident at a grazing angle to the surface and may be considered of no experimental interest. However, it should be noted that there have been recent experimental measurements, primarily observing scattering by surface defects, which have been carried out under conditions in which grazing angle final beams have been observed, ${ }^{14,15}$ so such a case should not be completely dismissed out of hand. Finally, it should be noted that if the parallel momentum $|\Delta K+N|>\sqrt{\Delta E-\left|\epsilon_{n}\right|}$, i.e., if the parallel wave vector is above the threshold for entering the bound state, there are no solutions of interest of Eq. (9).

Shown in Fig. 1 is a calculation for a typical scattering configuration, in this case corresponding to a phonon at the surface Brillouin zone boundary for $\mathrm{He}$ scattering on $\mathrm{Cu}(001)\langle 110\rangle, \quad$ in which $\Delta E=17 \mathrm{meV}, \quad \Delta K=-1.2$ 
$\AA^{-1}, N=0$, and $\epsilon_{0}=4.6 \mathrm{meV}$. Figure 1 (a) is a plot of $k_{i}$ as a function of $\theta_{i}$ from Eqs. (3) or (9). Both the minimum point at $\theta_{\min }=14.63^{\circ}$ with $k_{\min }=4.87 \AA^{-1}\left(E_{\min }\right.$ $=12.4 \mathrm{meV}$ ) and the maximum point at $\theta_{\max }=90^{\circ}$ with $k_{\max }=10.26 \AA^{-1} \quad\left(E_{\max }=55.03 \mathrm{meV}\right)$ exhibit the singular behavior, which appears here as a vanishing slope. Figure 1(b) shows $d k_{i} / d \theta_{i}$ as a function of $\theta_{i}$, i.e., the inverse of the Jacobian derivative of Eq. (8), and the singular points are exhibited clearly as zeros of this derivative. Thus for these fixed values of phonon energy and momentum, a $\mathrm{He}$ atom beam incident with an energy of $12.4 \mathrm{meV}$ at an angle of $14.63^{\circ}$ with respect to the normal will exhibit focusing into the bound state. These are values readily obtainable in $\mathrm{He}$ atom scattering experiments. It is clear from Fig. 1(a) that the FS condition at $\theta_{i}=\theta_{\min }$ is a strong focusing effect because the slope of the $k_{i}$ versus $\theta_{i}$ curve is very close to vanishing over a large range of angles near $\theta_{\min }$.

One can also consider the case which is just the opposite of Eq. (6) for which the beam is very well defined in its angular distribution but there is a spread of incident wave vectors. In this case the incident distribution function has the following approximate form

$$
\rho_{\mathbf{k}_{i 0}}\left(\mathbf{k}_{i}\right)=\delta\left(\theta_{i}-\theta_{i}\left(k_{i}\right)\right) f\left(k_{i}\right) .
$$

The partial sticking coefficient, corresponding to Eq. (6) now becomes

$$
\begin{aligned}
& \sum\left(\mathbf{k}_{i 0} ; n, N, \Delta E, \Delta K\right) \\
& \quad=\int_{\Delta \theta_{i}} d \theta_{i}\left|\frac{d k_{i}}{d \theta_{i}}\right|_{k_{i}^{*}} f\left(k_{i}^{*}\right) \sigma\left(\mathbf{k}_{i} ; n, N, \Delta E, \Delta K\right),
\end{aligned}
$$

where $k_{i}^{*}\left(\theta_{i}\right)$ is the value given by the resonance equation (3) for specified $\Delta E$ and $\Delta K$. In this case the singularity would arise from the vanishing of the numerator of the derivative of Eq. (8) which leads to

$$
\cos ^{2} \theta_{i}=\frac{(\Delta K+N) \sin \theta_{i}}{k_{i}}=\frac{(\Delta K+N)^{2}}{-\Delta E+\left|\epsilon_{n}\right|} .
$$

Since, for a sticking event, $\Delta E$ must be positive and larger than $\left|\epsilon_{n}\right|$, Eq. (15) would imply unphysical values of $\theta_{i}$ and consequently focusing does not occur in this case. Equation (15) can be satisfied as $\theta_{i} \rightarrow 90^{\circ}$ for large $k_{i}$, but this case is of no importance to the present sticking problem.
However, for scattering into continuum states, the counterpart of Eq. (15) does give rise to focusing at physically achievable incident angles. This particular case of focusing in continuum state scattering has been previously studied and it is called the inelastic critical kinematic effect. ${ }^{9}$ If $\Delta E$ and $\Delta K$ are zero, this same focusing is manifest in the elastic scattering where it is called elastic critical kinematic scattering. ${ }^{7}$

In this paper it is shown that focusing processes, of which several specific cases are now well documented for continuum state molecular- and atom-surface scattering, can be extended to the case of scattering into the negative energy bound states where they affect the measured value of the sticking coefficient or can enhance the intensity of scattering into a particular bound state. The focused sticking presented here can be directly related to similar effects that have been observed in continuum state scattering, hence giving assurance that it will be observed, and can cause significant enhancement of the sticking coefficient under circumstances in which a single phonon mode of energy $\Delta E$ and parallel momentum $\Delta K$ makes an important contribution to the sticking. Certain continuum-to-bound state transitions are directly observable as resonances in the continuum scattering, such as phonon assisted selective adsorption processes, ${ }^{10}$ and FS may be utilized to enhance these processes. Similarly, since FS occurs at the threshold for which a particular phonon of energy $\Delta E$ and parallel momentum $\Delta K$ causes a transition to a well-defined negative energy sticking state, the incident energy and angle can be "tuned" to focus on individual phonons in the surface spectral density. This focusing should be particularly applicable to the case of localized surface modes due to adsorbates and adsorbate layers on the surface.

This focused sticking is not a result of the dynamics of the scattering process, and hence does not depend on details of the interaction potential. Instead, it occurs at particular combinations of incident angle and energy for which the angular spread of the incident beam wave packet is focused into a single quantum transition. This phenomenon should be observable, since its counterparts in continuum state scattering have already been experimentally observed.

This work was supported in part by DGICYT (Spain) under Contract No. PB95-0071 and by the Division of Materials Research of the NSF and the Division of Basic Energy Sciences of the DOE.
${ }^{1}$ G. P. Brivio and T. B. Grimley, Surf. Sci. Rep. 17, 1 (1993).

${ }^{2}$ J. Böheim, Surf. Sci. 148, 463 (1984).

${ }^{3}$ M. D. Stiles and J. W. Wilkins, Phys. Rev. Lett. 54, 595 (1985)

${ }^{4}$ S. Andersson, L. Wilzen, M. Persson, and J. Harris, Phys. Rev. B 40, 8146 (1989).

${ }^{5}$ G. Armand and J. R. Manson, Phys. Rev. B 43, 14371 (1991).

${ }^{6}$ G. Benedek, Phys. Rev. Lett. 35, 234 (1975).
${ }^{7}$ M. Hernandez, S. Miret-Artés, P. Villarreal, and G.DelgadoBarrio, Surf. Sci. 251/252, 369 (1991); 274, 21 (1992).

${ }^{8}$ G. Benedek and S. Miret-Artés, Surf. Sci. Lett. 339, L935 (1995); S. Miret-Artés, ibid. 366, L735 (1996).

${ }^{9}$ S. Miret-Artés, Phys. Rev. B 60, 1547 (1999).

${ }^{10}$ D. Evans, V. Celli, G. Benedek, J. P. Toennies, and R. B. Doak, Phys. Rev. Lett. 50, 1854 (1983). 
${ }^{11}$ G. Benedek, R. Gerlach, A. Glebov, G. Lange, S. Miret-Artés, J. G. Skofronick, and J. P. Toennies, Phys. Rev. B 53, 11211 (1996); M. F. Bertino, S. Miret-Artés, J. P. Toennies, and G. Benedek, ibid. 56, 9964 (1997); M. F. Bertino, S. Miret-Artés, and J. P. Toennies, Chem. Phys. Lett. 287, 663 (1998).

${ }^{12}$ A. Glebov, J. R. Manson, S. Miret-Artés, J. G. Skofronick, and J. P. Toennies, Phys. Rev. B 57, R9455 (1998); 59, 7767 (1999).
${ }^{13}$ H. Hoinkes and H. Wilsch, in Helium Atom Scattering from Surfaces, edited by E. Hulpke, Springer Series in Surface Sciences 27 (Springer Verlag, Heidelberg, 1992), p. 113.

${ }^{14}$ A. Glebov, J. R. Manson, J. G. Skofronick, and J. P. Toennies, Phys. Rev. Lett. 78, 1508 (1997).

${ }^{15}$ D. Farias, M. Patting, K.-H. Rieder, and J. R. Manson (to be published). 